\title{
The Spectral Evolution of Post-AGB Stars
}

\author{
R.D. Oudmaijer ${ }^{1,2}$, P.A.M. van Hoof ${ }^{2}$ and L.B.F.M. Waters ${ }^{3,4}$ \\ ${ }^{1}$ Imperial College, London; ${ }^{2}$ Kapteyn Institute Groningen; \\ ${ }^{3}$ Astronomical Institute 'Anton Pannekoek', Amsterdam; ${ }^{4}$ SRON, Groningen
}

We have performed a parameter study of the spectral evolution of a typical post-AGB star with particular emphasis on the evolution of the IRAS colours. The models are based on the latest evolutionary tracks by Blöcker (1995, A\&A 297, 727 and A\&A 299, 755), which are used to define the evolutionary rate and the mass loss history of the central star. The resulting model for the post-AGB star is then used to calculate the spectral evolution with the photo-ionization code CLOUDY (Ferland 1993, Univ. of Kentucky, Int. Report), which includes dust in the radiative transfer.

We considered different assumptions for the dust properties and dust formation. It was found that by varying certain input parameters in a reasonable way, entirely different paths are followed in the IRAS colour-colour diagram. One of the most important parameters that governs the infrared evolution is the effect of the increasing temperature of the central star on the expanding shell, resulting in clock-wise loops in the diagram (Fig.1), instead of the conventional counter-clock wise loops (e.g. Volk \& Kwok 1989, ApJ 342, 345).

Also, the dust properties and the moment of cessation of the heavy AGB mass loss have an important effect. The model tracks show that objects which occupy the same location in the IRAS colour-colour diagram can have a different evolutionary past, and therefore the position in the diagram alone can not a priori give a unique determination of the evolutionary status of an object. A more extensive account of this work is given in van Hoof, Oudmaijer \& Waters (1997, MNRAS submitted).

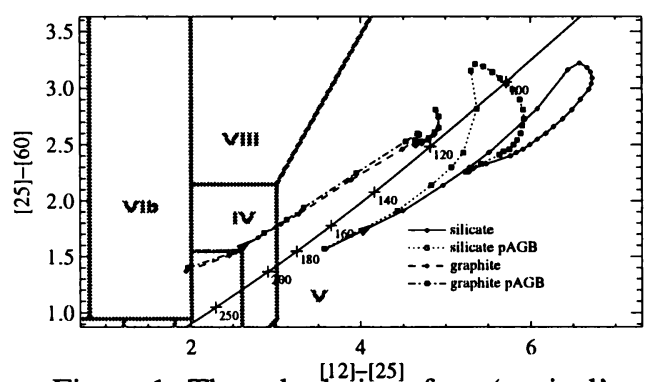

Figure 1: The calculations for a 'typical' post-AGB star (core mass $0.605 \mathrm{M}_{\odot}$, initial mass $3 \mathrm{M}_{\odot}$ ) are presented. The calculations start at $\mathrm{T}_{\text {eff }} \approx 6000 \mathrm{~K}$, the start of the post-AGB phase, and end at $T_{\text {eff }}=35000 \mathrm{~K}$. We present 4 cases: a silicate and a graphite model, both with and without post-AGB dust formation. 